\title{
EVOLUCION EN LAS CONCEPCIONES SOBRE EVALUACION EN ESTUDIANTES DEL PROGRAMA DE MAESTRIA EN DOCENCIA DE LA QUÍMICA
}

\author{
Luis E. Salcedo, \\ Lilia Reyes, \\ Andrés Perafán
}

\begin{abstract}
In this article we present the results of the application of a pedagogyca/ strategy to chemistry tea chers. This strategy was cre ated in order to permit the refiection upon the issues of evaluation in theory and practice. In this way, we can crea te real alternatives for transformation which contribute to an improvement of the quality of chemical education. Teachers are ab/e to change their perception of evaluation from a final accumulative proccess which centers attention only on the student to a concept of evaluation as a means of learning and improving the proccess of teaching. Through this strategy the teachers apply this vision to the development of chemical subjects based on a series of activities centered on problematic situations which evaluate throughout the proccess favoring a significant adquizition of chemistry
\end{abstract}

\section{RESUMEN}

En este artículo damos cuenta de la experimentación de una estrategia Pedagógicodidáctica aplicada a profesores de química en servicio activo creada para posibilitar espacios de reflexión sobre los problemas asociados a las concepciones y prácticas de evaluación y para vivenciar alternativas de transformación que contribuyan a mejorar la calidad de la educación en química. Los maestros logran cambiar la idea de evaluación como proceso terminal, acumulativo y centrado en el estudiante a una concepción de evaluación como medio de aprendizaje y mejora de la docencia. Aplican esta visión al desarrollo de temáticas en química, basadas en programas de actividades centradas en situaciones problemáticas, que evalúan a lo largo de todo el proceso, favoreciendo el aprendizaje significativo de la química.

Palabras claves: Evaluación, creencias, acciones, cambio, Docencia de la Química

\section{Introducción}

En un trabajo de investigación apoyado por COLCIENCIAS y la UPN real izamos un estudio interpretativo en torno a Las creencias que sobre la ciencia, la enseñanza y el aprendizaje mantienen algunos profesores de química (Salcedo y otros200l) de física (Perafán y otros 2001) y de biología (Reyes y otros 2001) que laboran en tres instituciones de educación secundaria del Distrito Capital. Al analizar las acciones cotidianas de estos maestros en el aula encontramos, por una parte, que en sus actividades de evaluación se hacían explícitas muchas de sus creencias sobre la ciencia, la enseñanza y el aprendizaje y por otra, que los cambios sugeridos en las disposiciones legales sobre logros e

\footnotetext{
*Profesores Universidad Pedagógica Nacional. Programa Interinstitucional de Doctorado en Educación Area Educación en Ciencias Naturales, Centro de Investigaciones CIUP.
} 
indicadores de logro no encuentran en la práctica una aplicación coherente y se han generado situaciones problemáticas que ameritan un trabajo investigativo en este campo. En otra investigación, apoyada por el CIUP, caracterizamos las concepciones que sobre evaluación mantienen los profesores formadores de maestros de química encontrando que la evaluación no es considerada como un medio de aprendizaje y mejora de a docencia y sí como una actividad para la constatación terminal y acumulativa de conocimientos en el estudiante, centrada en el manejo operatorio de conceptos químicos olvidando aspectos metodológicos y actitudinales (Salcedo y Villarreal 1999)

Como aporte a la problemática de la evaluación trabajada por otros investigadores (Alonso 1990 y 1992; Gil y Martínez torregrosa 1992; Rincón 1996; Valcarcel 1996) hemos vertido experimentando estrategias pedagógico-didácticas con profesores de ciencias experimentales y de química en particular con el fin de generar espacios de reflexión sobre los problemas allí detectados y plantear alternativas de solución a los mismos con la participación activa de los profesores de secundaria y de las instituciones formadoras de docentes.

\section{Referentes conceptuales}

Dado que en otros trabajos nos hemos referido a los aspectos centrales del modelo constructivista de evaluación (Salcedo y otros 1996; Salcedo y Villarreal 1999) aquí reiteramos solamente la idea central que durante todo el trabajo realizado cori los n'taestros nos sirvió como punto de apoyo para aportar al análisis de las situaciones problemáticas referidas a la evaluación y al análisis de concepciones alternativas para la transformación del pensamiento y las acciones de los participantes en el seminario. Insistimos en la idea de considerarla evaluación de la enseñanza y del aprendizaje de la química como un instrumento de aprendizaje y de mejora de la docencia; instrumento de aprendizaje en la medida que las actividades de evaluación favorecen el aprendizaje significativo de la química e instrumento de mejora de la enseñanza por cuanto aporta al conocimiento de los logros y deficiencias del trabajo docente.

\section{ASPECTOS METODOLOGICOS}

El trabajo se realizó en dos etapas a saber:

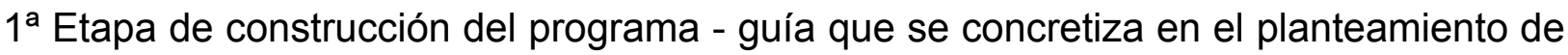
una serie de actividades con las cuales dinamizamos la reflexión de los maestros y aportamos nuestros conocimientos para someternos al análisis critico de los participantes y así favorecer las decisiones de cambio. Esta etapa incluye también la preparación de documentos teóricos para ser sometidos al análisis crítico de los participantes y a través de ellos aportar a la generación de otras visiones.

$2^{a}$ Etapa de experimentación del programa-guía con los estudiantes del programa de maestría en docencia de la química en el seminario de conceptos químicos e implicaciones didácticas. EL desarrollo de las actividades se llevó a cabo en tres sesiones de 12 horas cada una. Para sistematizar la información, los investigadores organizaron para cada profesor una carpeta en la cual se han llevado los registros correspondientes a los trabajos escritos presentados por los maestros durante el desarrollo de cada una de las actividades del programa - guía y los proyectos finales presentados. 


\section{Instrumentos utilizados}

Los instrumentos utilizados para recolectar la información se elaboraron buscando no solo verificar la ausencia en el pensamiento y acción de los profesores de características propias de la nueva evaluación, sino pretendiendo también caracterizar dicho pensamiento y actuación docente acerca de la evaluación, para mostrar que las ideas y comportamiento de los profesores son coherentes o no, al paradigma de enseñanza de transmisión - asimilación. Estos instrumentos están integrados a las actividades del programa-guía desarrolladas por los participantes. Además, cada actividad del programa guía se convierte en un instrumento de recolección de información la cual se obtiene durante todo el proceso de interacción con los participantes lo cual permite tener elementos sobre los cambios que se están produciendo en los participantes.

\section{¿Cómo se sistematizaron los cambios en las concepciones?}

Se realizó un diagnóstico para caracterizar las concepciones de los participantes sobre la evaluación cuyos resultados quedaron consignados para cada maestro en su carpeta de seguimiento y sirvieron a la vez como instrumento de intervención didáctica a través del análisis critico de las mismas a la luz del modelo de evaluación propuesto, con el fin de aportar nuevas ideas que ampliaran las visiones iniciales. Durante todo el proceso de interacción entre los participantes y los investigadores en cada una de las actividades desarrolladas se recogió información escrita y oral que quedó consignada en papel. Los avances y los retrocesos en la transformación de las ideas de los maestros se hacían explícitos a través de la participación de los mismos en cada una de las actividades desarrolladas primero a nivel individual y por escrito, luego las sesiones de intercambio a nivel de los pequeños grupos y finalmente en el trabajo de plenaria. Adicionalmente, se recogió información a partir de las tareas propuestas para realizar fuera del aula y en la presentación oral y escrita de las propuestas de evaluación formuladas por los maestros. En búsqueda de coherencia entre la idea fundamental de hacer de la evaluación un instrumento de aprendizaje y de mejora de la docencia y la 'medida' de los cambios producidos en los participantes no hemos dejado para el final la evaluación de los logros y limitaciones; hemos estado atentos a llevar registros para cada una de las actividades del programa-guía; por supuesto que también hemos tenido en cuenta los productos resultantes en los momentos claves pero no han sido estos los únicos criterios de evaluación de todo el trabajo llevado acabo.

A continuación se presentan los resultados obtenidos al aplicar el programa -guía. Se incluyen las actividades realizadas con los profesores con sus comentarios; las respuestas dadas por los profesores y las actuaciones de los investigadores en estos procesos de intervención didáctica y algunos productos elaborados por los maestros como elementos que aportan evidencias de los cambios logrados. 


\section{RESULTADOS Y ANÁLISIS PROGRAMA-GUÍA}

\section{Conceptos químicos e implicaciones didácticas vs. Evaluación}

\section{Introducción}

\section{Apreciados compañeros}

Reciban un cordial saludo y una invitación para que en este espacio académico, a partir de nuestros conocimientos y experiencias podamos realizar un análisis conjunto de los principales problemas que se presentan en la enseñanza y el aprendizaje de la química, en particular, los problemas referidos a la enseñanza y al aprendizaje de conceptos químicos y sus posibilidades de evaluación.

Aspiramos poder aportar una metodología de trabajo que logre sintetizar, ojalá con el apoyo de la historia de la química, un buen conocimiento de esta disciplina en función de la comprensión de los problemas que su enseñanza y su aprendizaje generan en los estudiantes de los diferentes niveles de nuestro sistema educativo y de sus implicaciones en los procesos evaluativos

En esta sesión trabajaremos aspectos relacionados con nuestras intencionalidades como enseñantes de la química y los requerimientos en términos de formación profesional para un trabajo docente de calidad. En este contexto ampliaremos nuestra visión de lo que significa saber química. Tomaremos como conceptos de referencia aquellos que forman parte obligada de la mayoría de cursos de química con la intención de mejorar nuestra comprensión para conocer mejor las dificultades de aprendizaje asociadas a dichas temáticas en procura de mejorar nuestros proyectos de aula. Estaremos refiriéndonos todo el tiempo a la problemática de la evaluación y a la posibilidad de hacer de ella un instrumento de aprendizaje y de mejora de la docencia.

Los invitamos pues a desarrollar con mucho entusiasmo y compromiso las siguientes actividades:

Actividad No. 1. En ferina individual y por escrito responda la siguiente cuestión: ¿Para qué enseña usted química?

Actividad No. 2. Analice en pequeños grupos ( 3 a 4 ) las respuestas dadas y elaboren una propuesta de grupo para ser presentado en el plenario.

Actividad No. 3. Con la orientación del investigador intercambiar a nivel general las propuestas de grupo anteriormente elaboradas. Definir los puntos de acuerdo y aquellos en los que hay desacuerdos

Comentario: En este grupo de participantes hemos querido plantear la problemática de la evaluación en un contexto más amplio al puramente disciplinar para así darle una dimensión más filosófica a las acciones docentes y en particular a las acciones evaluativas. Pretendemos que los participantes ubiquen la evaluación en todo el proceso de enseñanza y aprendizaje.

Las respuestas de los profesores se refieren en su mayoría a aspectos referidos a la disciplina centrando su atención en la comprensión de las teorías, las leyes, los principios 
de la química. Especial mención hacen al aprendizaje de conceptos y su aplicación en la vida diaria.

Se comparte con el grupo un documento escrito elaborado por los investigadores para aportar a la contrastación de ideas que propone finalidades alternativas a las tradicionalmente aceptadas. Se realizó una invitación a los docentes a tomar cada uno de estos enunciados como situaciones problemáticas a resolver más que como frases externas que no se corresponden con nuestras actuaciones. La idea es seguir pensando sobre el significado y tas implicaciones que dichos enunciados tendrían para nuestra actividad educadora. Así por ejemplo, cuando decimos que la educación en química debería aportar al desarrollo de la autonomía del joven ¿qué significado tiene para cada uno de nosotros? y ¿cómo hacer de nuestra clase un espacio propicio para el desarrollo de dicha categoría en nuestros estudiantes?

En la interacción llevada a cabo quedó claro que no podemos reducir las intencionalidades de nuestras acciones docentes a lo puramente disciplinar sino que además debe trascender a aquellos aspectos de la formación del sujeto que son fundamentales para su pleno desarrollo y allí estaría contenido explícitamente el papel formativo de la química. De todas maneras insistimos en la necesidad de dejar el terna de las intencionalidades como una preocupación intelectual para seguir pensando; no agotar el tema con lo planteado en el documento.

Teniendo como referencia las grandes intencionalidades con las cuales abordamos nuestro trabajo vamos ahora a pensar en la formación profesional que deberíamos tener para poder aproximarnos al logro de dichas finalidades.

Actividad No. 4. En forma individual y por escrito responda la siguiente cuestión ¿Qué debería saber y saber hacer un profesor de química para cumplir exitosamente con el logro de las finalidades descritas en la actividad anterior? Discutir luego en pequeños grupos y hacer una presentación al plenario. El investigador orientará la actividad y realizará los aportes respectivos.

Comentario: se pretende con esta actividad abrir un espacio de reflexión para que sea el mismo maestro quien defina los rasgos de su formación que aproximarían en la acción, al logro de lo que se quiere con la educación en química.

De esta actividad surgió un listado muy amplio de competencias profesionales que sirvió como soporte para tomar conciencia de la complejidad del trabajo docente y de la necesidad de conformar grupos de trabajo tanto a nivel institucional como interinstitucional para poder llevar a cabo con éxito, las acciones de innovación y/o investigación por parte de los maestros. La mayoría de los profesores están de acuerdo que uno de los conocimientos fundamentales es el referido a la materia a enseñar; se afirma también la necesidad de tener conocimientos de disciplinas tales como: filosofía, antropología, pedagogía, sicología, sociología y didáctica pero lo más importante es poder traducir esos saberes en acciones concretas de planeación, orientación y evaluación del aprendizaje de los estudiantes. Un buen profesor debería también participar en proyectos de innovación o de investigación.

En estas interacciones los investigadores enfatizan la idea de incorporar la evaluación a todo el proceso de planeación y ejecución del trabajo en el aula para hacer de ella un instrumento de aprendizaje y mejora de la enseñanza. 
Actividad No. 5. ¿Es posible que un profesor como usted tenga toda la preparación que se ha señalado anteriormente? Hacer los comentarios del caso

Comentario: La idea aquí es posibilitar una mirada equilibrada de las posibilidades y las limitaciones que nuestro trabajo educativo nos ofrece. Reforzar la idea de la necesidad de mantener una actitud de permanente preparación y por otra la necesidad de trabajar en equipos.

Las respuestas de los profesores se dan en uno y en otro sentido. Los investigadores invitan a desarrollar una concepción optimista pero al mismo tiempo realista enfatizando la necesidad de realizar un trabajo en equipo y de mantener una actitud de formación permanente.

Actividad No. 6. Por escrito responda ¿qué significa para usted conocer la materia a enseñar? Intercambie sus ideas con los compañeros en grupos y realicen una presentación en la plenaria.

Comentario: con esta actividad se quiere posibilitar una reflexión en torno a una temática muy importante para el desempeño de un buen profesor de química. Si bien todos los maestros están de acuerdo con la importancia de saber la materia a enseñar, no existe tal consenso a la hora de definirlo que significa saber la materia.

Al respecto los profesores señalan que un buen conocimiento de la materia implica saber las teorías, las leyes, los principios de la disciplina. No se refieren explícitamente a aspectos tales como conocer los problemas que originaron los conocimientos de que disponemos hoy dia. Tampoco mencionan la necesidad de conocer las metodologías con las cuales los científicos de la química abordaron dichos problemas. Dejan de lado el conocimiento de las relaciones entre la disciplina, los desarrollos tecnológicos de la misma y su implicaciones sociales, económicas y políticas. Tampoco se refieren al conocimiento de temas frontera que amplían la visión reduccionista de la química. Poco mencionan la importancia de saber seleccionar los contenidos y darles el tratamiento didáctico necesario para responder a las necesidades de los estudiantes.

En el plenario se realizó un análisis crítico a las respuestas y se aportaron otros elementos que ampliaron la visión inicial. Para complementar el trabajo se entregó para su lectura el articulo de Daniel Gil "Lo que debe saber y saber hacer un profesor de ciencias para realizar un trabajo docente de calidad' publicado en la Revista de Enseñanza de las Ciencias. Se solicitó a los participantes confrontar sus respuestas con lo planteado en el documento y realizar los comentarios del caso. En particular se solicitó realizar un análisis al bloque de conocimientos relacionado con el saber evaluar y las implicaciones con el conocimiento de la materia a enseñar.

Actividad No. 7. En este contexto de definición de las competencias deseables para realizar una docencia de calidad en química el tema de la evaluación es fundamental. Abramos un espacio de discusión y análisis para repensar nuestras concepciones y acciones en este campo y tomar decisiones para introducir los cambios que consideremos necesarios. En forma individual y por escrito responda la siguiente cuestión: ¿con que fines realiza Ud. la evaluación?

Las respuestas de los profesores son: 
Fines de la evaluación —Muestra 22 profesores-

\begin{tabular}{|l|c|}
\hline CATEGORIAS & Porcentajes \\
\hline $\begin{array}{l}\text { a. Establecer dificultades, deficiencias } \\
\text { De estudiantes }\end{array}$ & $27,3 \%$ \\
\hline b. Medir desempeño docente - metodologías & $40,9 \%$ \\
\hline c. Revisar reestructuración de conceptos & $36,4 \%$ \\
\hline d. Verificar “asimilación" de temas propuestos & 36,4 \\
\hline $\begin{array}{l}\text { e. Reconocer relaciones que los estudiantes } \\
\text { Establecen entre conceptos y temas }\end{array}$ & $4,5 \%$ \\
\hline f. Retroalimentación (aula-maestro-alumno) & $9,0 \%$ \\
\hline $\begin{array}{l}\text { g. Capacidad de interpretación y solución de } \\
\text { Situaciones problemáticas }\end{array}$ & $9,0 \%$ \\
\hline h. Exigencia institucional & $4,5 \%$ \\
\hline $\begin{array}{l}\text { i. Buscar metodologías para mejorar la } \\
\text { comprensión }\end{array}$ & $18,1 \%$ \\
\hline j. Identificar habilidades de los estudiantes & $4,5 \%$ \\
\hline k. Identificar el lenguaje de los estudiantes & $4,5 \%$ \\
\hline
\end{tabular}

Con este grupo de maestros se obtienen respuestas más próximas al modelo de evaluación como instrumento de aprendizaje y mejora de la docencia ya que se hace referencia a los fines de la evaluación no sólo para conocer lo que ocurre con los estudiantes sino también lo que pasa con los profesores y las metodologías utilizadas. Los profesores manifestaron en un alto porcentaje $(40,900)$ que es útil para medir tanto el desempeño docente como las metodologias empleadas. También es significativo el interés por revisar la reestructuración de conceptos en los estudiantes (36,4\%). De este modo se refleja que la perciben corno un instrumento de ayuda para favorecer un aprendizaje significativo (ver items a, e, q, i, j, k). Sin embargo, no parece importante SU función como indicador de aprendizaje dirigido a los estudiantes para su conscientización frente al proceso de aprendizaje, mencionándose a este respecto la categoría retroalimentación (aula-maestro-alumno) con apenas un $9 \%$.

Los investigadores valorando las respuestas aportan al grupo la idea fundamental de considerar la evaluación como un instrumento de aprendizaje; es decir como un proceso continuo y permanente que le permite al estudiante tornar plena conciencia de sus avances y dificultades para tomar las decisiones de acción que le permitan progresar intelectual, moral, afectiva y humanamente en búsqueda de su pleno desarrollo. Se plantea también la idea de darle a la evaluación una función referida a la mejora de la docencia en el sentido que los profesores al evaluar su trabajo permanente y sistemáticamente van a tener mayores posibilidades de conocer sus logros y dificultades que les permitan producir los cambios que contribuyan a realizar acciones docentes de calidad. La ampliación de la evaluación al funcionamiento de la institución favorece también procesos de cambio para mejorar la organización escolar. Se retorna la idea de ampliar la evaluación más allá de aspectos conceptuales para tener en cuenta lo procedimental (Payá 1991) y lo actitudinal (Shcibeci 1984), es decir, para favorecer aprendizajes significativos y de mejoramiento de la comprensión de las relaciones entre la ciencia química, la tecnología y la sociedad (Vilches 1991, Beltrán y otros 1997)

Para continuar el análisis de los aspectos centrales de la evaluación se realiza la siguiente actividad. 
Actividad No. 8. En forma individual escriba las características de la evaluación que Ud. practica.

\section{Caracteristicas de la evaluación}

-Muestra 22 profesores-.

\begin{tabular}{|l|c|}
\hline CATEGORIAS & Porcentajes \\
\hline $\begin{array}{l}\text { a. Análisis y resolución de situaciones } \\
\text { Problemáticas )cotidianas o teóricas) }\end{array}$ & $18,1 \%$ \\
\hline b. Detección de manejo conceptual & $18,1 \%$ \\
\hline $\begin{array}{l}\text { c. Identificación de situaciones conflictivas } \\
\text { En lo conceptual o lo metodológico }\end{array}$ & $4,5 \%$ \\
\hline d. Continúa & $31,8 \%$ \\
\hline e. Grupal, individual y autoevaluación & $18,1 \%$ \\
\hline f. Claras, con preguntas cerradas y abiertas & $4,5 \%$ \\
\hline g. Cortas & $9,0 \%$ \\
\hline h. Con un punto de memoria & $4,5 \%$ \\
\hline i. Objetiva & $9,0 \%$ \\
\hline j. Como medida represiva & $4,5 \%$ \\
\hline k. De tipo cualitativo & $9,0 \%$ \\
\hline I. Cuestionarios, trabajos, exposiciones & $22,7 \%$ \\
\hline $\begin{array}{l}\text { m. Persigue que el alumno construya modelos } \\
\text { Nuevos }\end{array}$ & $4,5 \%$ \\
\hline $\begin{array}{l}\text { n. Genera información para determinar } \\
\text { Una decisión pedagógica }\end{array}$ & $4,5 \%$ \\
\hline o. Constructivista / mapas conceptuales & $9,0 \%$ \\
\hline p. De procesos & $18,1 \%$ \\
\hline $\begin{array}{l}\text { q. Tienen en cuenta aspectos procedimentales } \\
\text { Actitudinales y conceptuales }\end{array}$ & $4,5 \%$ \\
\hline
\end{tabular}

En cuanto a las características de la evaluación, la categoría en la que hay más consenso es la señalada en el ítem (31 8\%); en general las apreciaciones se refieren a propiciar a un aprendizaje significativo (ver ítems $a, b, c, e, f, I, m, n, o, p, q, r)$. Entre otros, puede servir para un manejo de la "disciplina" (categoría k, como medida represiva. $4.5 \%$ ) o para perpetuar un aprendizaje memorístico (i, con un ponto de memoria. 4,5\%). En este aspecto se nota que la evaluación aunque pretende generar un acompañamiento en los procesos de aprendizaje tanto para estudiantes como para maestros, se convierte por su intencionalidad en un indicador de aprendizaje e instrumento de ayuda para mejorar su desempeño, más para los docentes que para los estudiantes. Se enfatiza en lo que el maestro pretende construir en los estudiantes y se pierde de este modo el otro sentido estudiante-maestro, de la función reguladora de la evaluación; no se percibe preocupación por la generación de una conciencia en el estudiante de sus propios avances, dificultades y necesidades.

Los investigadores resaltan las ideas relacionadas a la comprensión de la evaluación corno un proceso continuo, centrado en la resolución de problemas y en manejos conceptuales, pero advierten que precisamente la resolución de problemas es una de las estrategias más adecuadas no sólo para el manejo conceptual sino también para el procedimental y actitudinal. También se destaca la idea de la evaluación no sólo individual sino grupal y la autoevaluación como características importantes a tener en cuenta. 
Actividad No. 9. De manera individual y por escrito responda ¿En que aspectos enfatiza a la hora de evaluar? ¿qué porcentaje otorga a cada aspecto de acuerdo a la importancia que tiene para Ud.

Aspectos a evaluar -Muestra 22 profesores-.

\begin{tabular}{|c|c|c|}
\hline CATEGORIAS & \#RESP. & $\%$ \\
\hline a. Contenidos conceptuales & 11 & $50,0 \%$ \\
\hline b. Contenidos actitudinales - sociovalorativos & 12 & $54,5 \%$ \\
\hline c. Contenidos procedimentales - comunicativos & 15 & $68,2 \%$ \\
\hline d. Conocimientos teóricos & 9 & $40,9 \%$ \\
\hline e. Niveles de progreso - reestructuración & 1 & $4,5 \%$ \\
\hline f. Competencia interpretativa & 4 & $18,1 \%$ \\
\hline g. Conceptos o símbolos memorísticos & 2 & $9,0 \%$ \\
\hline h. Capacidad de análisis o cuestionamiento del alumno & 4 & $18,1 \%$ \\
\hline i. Creatividad & 2 & $9,0 \%$ \\
\hline j. Manejo de conceptos en hechos reales o cotidianidad & 3 & $13,6 \%$ \\
\hline $\begin{array}{l}\text { k. Habilidad para resolver ejercicios de tipo matemático } \\
\text { Aplicados a la química }\end{array}$ & 1 & $4,5 \%$ \\
\hline I. Competencia argumentativa & 3 & $13,6 \%$ \\
\hline m. Competencia propositiva & 3 & $13,6 \%$ \\
\hline n. Saber ser & 1 & $4,5 \%$ \\
\hline o. Asistencia & 1 & $4,5 \%$ \\
\hline p. Generación de conceptos propios & 1 & $4,5 \%$ \\
\hline
\end{tabular}

Participación porcentual de los aspectos enunciados en el total de la evaluación.

\begin{tabular}{|l|c|c|c|}
\hline CATEGORIAS & $\begin{array}{l}\text { PARTIC } \\
\% \\
\text { MAXIMA }\end{array}$ & $\begin{array}{l}\text { PARTIC. } \\
\% \\
\text { MÍNIMA }\end{array}$ & $\begin{array}{l}\text { PARTIC. \% } \\
\text { PROMEDIO }\end{array}$ \\
\hline a. Contenidos conceptuales & $50,0 \%$ & $20,0 \%$ & $34,0 \%$ \\
\hline b. Contenidos actitudinales - sociovalorativos & $33,3 \%$ & $5,0 \%$ & $26,4 \%$ \\
\hline c. Contenidos procedimentales - comunicativos & $50,0 \%$ & $20,0 \%$ & $36,6 \%$ \\
\hline d. Conocimientos teóricos & $60,0 \%$ & $20,0 \%$ & $36,6 \%$ \\
\hline e. Niveles de progreso - reestructuración & - & - & $20,0 \%$ \\
\hline f. Competencia interpretativa & $30,0 \%$ & $20,0 \%$ & $34,1 \%$ \\
\hline g. Conceptos o símbolos memorísticos & - & - & - \\
\hline h. Capacidad de análisis o cuestionamiento & $50,0 \%$ & $25,0 \%$ & $36,1 \%$ \\
\hline i. Creatividad & $50,0 \%$ & $20,0 \%$ & $35,0 \%$ \\
\hline j. Manejo de conceptos en hechos reales & $50,0 \%$ & $20,0 \%$ & $30,0 \%$ \\
\hline $\begin{array}{l}\text { k. Habilidad para resolver ejercicios de tipo } \\
\text { matemático aplicados a la química }\end{array}$ & - & - & $5,0 \%$ \\
\hline I. Competencia argumentativa & $30,0 \%$ & $20,0 \%$ & $25,0 \%$ \\
\hline m. Competencia propositiva & - & - & $40,0 \%$ \\
\hline n. Saber ser & - & - & $10,0 \%$ \\
\hline o. Asistencia & - & - & $10,0 \%$ \\
\hline p. Generación de conceptos propios & - & - & $20,0 \%$ \\
\hline q. No atribuyen porcentajes: 2 personas & & & \\
\hline r. No fue clara la respuesta: 1 persona & & & \\
\hline
\end{tabular}


Los aspectos a evaluar más mencionados son los conocimientos conceptuales o contenidos teóricos (los cuales suman 99,9\%), seguidos por los procedimentales comunicativos $(68,2 \%)$, y los contenidos actitudinales sociovalorativos $(54,5)$ La participación porcentual total de los aspectos evaluados según los docentes, corresponden a un $68,1 \%$ para la categoría conceptual - teórico. $36,6 \%$ para la procedimental - comunicativa, y $26,4 \%$ para la actitudinal-sociovalorativa. Las competencias también fueron bastante mencionadas: la competencia interpretativa con un $18,2 \%$, y las argumentativa y prepositiva con un $13,6 \%$ cada una. Tal vez podemos traducir en estas categorías otras expresadas como: capacidad de análisis o cuestionamiento del alumno (18,2\%) y manejo de conceptos en hechos reales o cotidianos $(13,6 \%)=$ competencias interpretativas; generación de conceptos propios $(4,5 \%)=$ competencia argumentativa; $y$ creatividad $(9,0 \%)=$ competencia propositiva. De este modo, sumando categorías similares, tendría finalmente una participación mayor la preocupación por la evaluación en competencias así: interpretativa 50\%, argumentativa $18,1 \%$ y prepositiva $22,6 \%$.

Los investigadores incorporan al debate las ideas relacionadas a la evaluación del aprendizaje significativo (Ausubel 1978) de la química el cual esta referido no solo a los aspectos conceptuales sino también procedimentales y actitudinales. Aportan la idea de considerar también el estudio de las implicaciones entre la ciencia química, la tecnología química y la sociedad como aspectos fundamentales del aprendizaje significativo. Queda aquí planteado el tema de las competencias que será abordado más adelante.

Actividad No. 10. Para aportar a una reflexión que tenga en cuenta las aplicaciones prácticas de estas posiciones solicitarnos a los profesores algunos exámenes aplicados a sus estudiantes con el fin de someterlos a un análisis crítico y poder utilizar dicho material como instrumento de intervención para hacer notar a los participantes las limitaciones de las pruebas escritas para la evaluación de los aprendizajes de los estudiantes.

Los investigadores realizaron el análisis a las pruebas aplicadas y los resultados fueron presentados y discutidos con los profesores participantes. Se gen eró una discusión que permitió aclarar muchos aspectos de las propuesta y su coherencia o no con el discurso de los profesores. En particular se mostró la fuerte tendencia a evaluar conceptos y a evaluar habilidades para la aplicación de fórmulas y/o definiciones, proponiendo relativamente pocas actividades para favorecer el aprendizaje significativo.

Los resultados del análisis de exámenes se muestran en la gráfica 1.

Gráfica 1. Análisis de exámenes de diferentes materias.

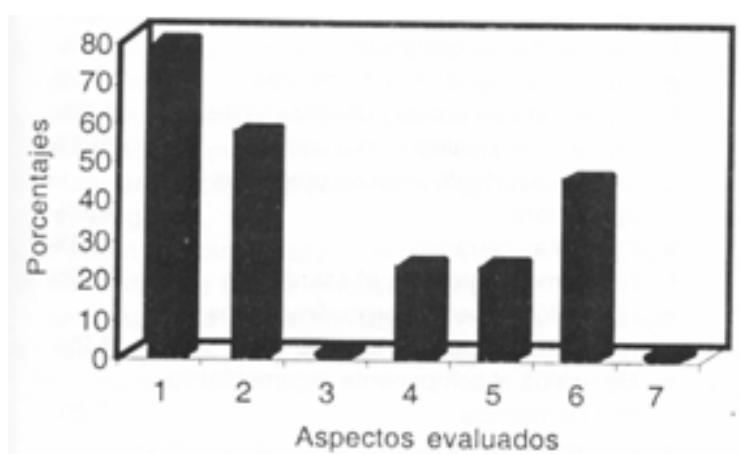

Preguntas sobre aspectos conceptuales de tipo memorístico.

2. Ejercicios de lápiz y papel cuya resolución involucra la aplicación de fórmulas mecánicamente

3. Actividades de autorregulación.

4. Actividades propias del trabajo cientifico

5. Actividades relacionadas con las relaciones $\mathrm{C} / \mathrm{T} / \mathrm{S}$

6. Actividades que sugieren aprendizaje significativo

7. Actividades sobre trabajo experimental 
Tal como se ilustra en la gráfica 1 en un $77,7 \%$ de las pruebas se encontraron actividades sobre aspectos conceptuales de tipo memorístico. Un $55.55 \%$ de las pruebas presenta ejercicios de lápiz y papel cuya respuesta depende de la utilización de una fórmula. Sin embargo en algunas pruebas se observan preguntas y actividades sobre el trabajo científico $(22.2 \%)$ y relaciones ciencia, tecnología y sociedad $(22.2 \%)$. Un $44.44 \%$ de pruebas contiene actividades en las que se evalúa el aprendizaje significativo de conceptos. Cabe anotar que en las pruebas no se realizan actividades de autorregulación e interregulación, ni tampoco actividades o preguntas sobre el trabajo experimental, lo cual sugiere evaluaciones de tipo tradicionalista en la mayoría de los profesores.

El análisis de estos resultados permite reiterarles a los profesores la invitación a realizar un esfuerzo conciente para buscar mayor coherencia respecto al aprendizaje significativo y las estrategias de evaluación.

Continuando con el análisis de situaciones concretas de evaluación que permiten tomar conciencia de los problemas y ampliar la visión sobre las posibles soluciones a los mismos ahora planteamos a los profesores participantes un ejercicio que consiste en analizar un examen de corte netamente tradicional con el fin de obtener sus impresiones y poder destacar en sus análisis aquellos aspectos que siguen siendo tradicionales y otros que seguramente aparecerán más ligados a la nueva concepción de evaluación.

Actividad No. 11. El siguiente examen fue propuesto al finalizar una temática sobre MCL y estequiometría a estudiantes de grado décimo (Tiempo $1 \frac{1}{2}$ horas). Por favor haga un análisis del mismo y realice los comentarios que considere oportunos respecto a la idoneidad del examen, posibles deficiencias, modificaciones, lo que le hace falta, lo que le añadiría, etc...

\section{QUÍMICA. EXAMEN FINAL SOBRE MOL - ESTEQUIOMETRIA}

1. Defina que es el MCL

2. Establezca las relaciones que el MOL tiene con el volumen molar y masa molar

3. La hidracina es usada para la combustión de cohetes al reaccionar con peróxido de hidrógeno, de acuerdo a la siguiente reacción

$\mathrm{N}_{2} \mathrm{H}_{4}+\mathrm{H}_{2} \mathrm{O}_{2}-\mathrm{HNO}_{3}+\mathrm{H}_{2} \mathrm{O}$

a. Balancee la ecuación

b. Si se tienen $100 \mathrm{~g}$ de hidracina y $100 \mathrm{~g}$ de peróxido, ¿cuántos g de ácido nítrico y agua se forman?

c. ¿Cuántos moles de reactivos sobran?

d. ¿Cuántas moléculas de agua pueden producirse al reaccionar exactamente 5 moles de peróxido?

4. ¿Qué cantidad de sustancia se encuentra contenida en un recipiente sellado de 4 litres que se encuentra a una temperatura de $100^{\circ} \mathrm{C}$ y 2 atm de presión y contiene dióxido de carbono $\left(\mathrm{CO}_{2}\right)$ ? 


\begin{tabular}{|l|c|}
\hline CATEGORIAS & Porcentajes \\
\hline a. Preguntas de tipo memorístico & $68,4 \%$ \\
\hline b. Enfatiza en contenidos conceptuales & $26,3 \%$ \\
\hline $\begin{array}{l}\text { c. Preguntas con resolución de tipo matemático } \\
\text { o con procedimientos algorítmicos }\end{array}$ & $63,1 \%$ \\
\hline d. La pregunta 4 no es pertinente & $5,2 \%$ \\
\hline e. No se preocupa por la interpretación del alumno & 26,3 \\
\hline f. Abarca mucho contenido & 5,2 \\
\hline g. No evalúa aspectos actitudinales & $10,5 \%$ \\
\hline h. No permite un análisis del estudiante & $5,2 \%$ \\
\hline i. No se contextualiza la evaluación & $26,3 \%$ \\
\hline j. La formulación de algunas preguntas no es clara & 5,2 \\
\hline k. Preguntas cerradas & $26,3 \%$ \\
\hline I. No es motivante para el estudiante & $21,0 \%$ \\
\hline $\begin{array}{l}\text { m. No evalúa aspectos procedimentales } \\
\text { ni la relación ciencia-técnica }\end{array}$ & $5,2 \%$ \\
\hline $\begin{array}{l}\text { n. No evalúa el componente argumentativo ni } \\
\text { de inferencia }\end{array}$ & $5,2 \%$ \\
\hline $\begin{array}{l}\text { o. No permite la evaluación de un aprendizaje } \\
\text { significativo }\end{array}$ & $5,2 \%$ \\
\hline p. Evalúa aprendizaje transmisionista & $10,5 \%$ \\
\hline
\end{tabular}

Las criticas a este examen se refieren en su mayoría a que las preguntas son de tipo memorístico $(68,4 \%)$, cerradas $(26.3 \%)$ y su resolución es de tipo mecánico $(63,1 \%)$. Se critica el énfasis en aspectos conceptuales y que las actividades en sí mismas no son motivantes para el estudiante. En general se es consciente de las principales limitaciones de la prueba lo que permite afirmar que los profesores participantes identifican los principales fallos de las evaluaciones tradicionales. Se critica la ausencia de actividades para cuya realización se requiere de cambios metodológicos y actitudinales y del análisis de las relaciones ciencia y tecnología, aspectos éstos fundamentales para el aprendizaje significativo.

La sugerencia más mencionada se refiere al planteamiento de situaciones problemáticas $(52,6 \%)$ para cuya comprensión y resolución se requeriría no sólo de manejos conceptuales sino también procedimentales y actitudinales. Se sugiere la necesidad de tener en cuenta las ideas previas de los estudiantes. Esto indica que la preocupación en la evaluación consiste en favorecer un aprendizaie significativo para casi todos los maestros consultados, lo cual nos aporta evidencias sobre los cambios que se van logrando en la comprensión de los aspectos a evaluar. Las demás categorías sugieren diversas ideas que enriquecen las posibles formas de evaluar.

Los investigadores valoran las respuestas y enfatizan las ideas que tienen relación con la producción de actividades que favorezcan el aprendizaje significativo dentro de un proceso amplio de resolución de problemas favoreciendo en todo caso aprendizajes conceptuales, metodológicos y actitudinales y el estudio permanente de las relaciones entre la ciencia química, la tecnología química y la sociedad.

Llegados a este punto, las diferentes actividades han permitido obtener información sobre las ideas de los profesores sobre aspectos claves de la evaluación. 
Paulatinamente, los investigadores han aportado al grupo a través de los análisis críticos a las respuestas conceptos fundamentales del modelo de evaluación como instrumento de aprendizaje y de mejora de la docencia con el fin de ir generando cambios en las ideas previas de los participantes. Ahora se abre el espacio para que todos puedan estudiar críticamente el documento "Concepciones y acciones de los profesores de química sobre la evaluación" (Salcedo y Villarreal 1999) y tengan así la oportunidad de confrontar sus ideas con las allí expuestas con el objetivo de contribuir a enriquecer la visión que hasta el momento se ha ido gestando.

Actividad No. 12. En forma individual realizar la lectura del documento "Concepciones y acciones de los profesores de química sobre la evaluación". Por escrito realizar un comentario sobre la concepción de evaluación que se propone. Argumentar sus puntos de acuerdo o desacuerdo. Destacar las funciones y características de la evaluación dentro de este modelo. Dar ejemplos de aplicación en temas específicos de la química.

\section{5}

Actividad No. 13. En pequeños grupos realizar una presentación del trabajo elaborado y preparar una presentación para el plenario.

Actividad No. 14. Con la orientación de los investigadores, llevar a cabo la sesión de confrontación de los resultados de las actividades anteriores. Realizar las aclaraciones, precisiones y aplicaciones para una mejor comprensión del modelo. Como tarea se solicita, aplicar el modelo a la definición de actividades concretas de evaluación en temas específicos de química.

Para continuar el trabajo de conceptualización y aplicación del modelo hemos introducido otras actividades ten-dientes a analizar el tema de las competencias, los logros y los indicadores de logro. Para tal efecto planeamos actividades que nos permitieran conocer las ideas previas sobre la temática y actividades para compartir con el grupo la visión de los investigadores.

Actividad No. 15. Por escrito y en forma individual responda la cuestión ¿qué es para Ud. una competencia? Comparta su posición con otros compañeros en pequeños grupos y luego presente una posición del grupo en la plenaria.

Las respuestas de los profesores presentan un amplio espectro de conceptos sin que haya una tendencia fuerte. Sin embargo la expresión "Saber hacer en un contexto" fue la concepción de competencia más nombrada. Otras concepciones con matices que van desde la explicitación de lo político, hasta la preocupación por la inserción a un sistema competitivo de los estudiantes, conforman el resto de las respuestas.

Sin entrar a criticar las diferentes posiciones se recomienda a los participantes realizar un trabajo de contextualizar sus ideas a propósito de la química para ir pasando de planteamientos generales a posiciones más relacionadas con la disciplina.

Actividad No. 16. En forma individual y por escrito responda: ¿qué competencias evalúa en sus clases y como las evalúa?

Las competencias más evaluadas según los docentes son la interpretativa y la argumentativa con un $80,0 \%$ cada una, seguidas por la prepositiva $(70,0 \%)$, comunicativa $(20,0 \%)$, de desarrollo - saber hacer $(20,0 \%)$, y la valorativa(15,0\%). 
El cómo evaluarlas se sugiere a través del planteamiento de situaciones problemáticas $(40,0 \%)$ y de las intervenciones verbales $(40,0 \%)$, además de la elaboración de resúmenes, ensayos (30\%), y de la realización de lecturas (científicas, gráficos, estadísticas 30,0\%). Estos medios a pesar de ser valiosos son los mismos de la enseñanza transmisionista. Otras propuestas incluyen: juegos, trabajos en grupo, realización de mapas, cuadros, críticas a lecturas, planteamiento de hipótesis y diseño experimental; permiten una mayor motivación, sensibilización y concientización de los estudiantes respecto a su aprendizaje y además exigen cambios en los imaginarios docentes.

Actividad No. 17. Por escrito responda: ¿Qué es para Ud. un logro y un indicador de logro?

Las percepciones de los profesores respecto a lo que es un logro, fundamentalmente evidencian una relación entre lo que el maestro desea generar y lo que el estudiante desarrolla en la práctica educativa. Se presenta una referencia permanente a los aspectos claves del aprendi zaje significativo. Sin embargo parece existir una cierta confusión entre considerar el logro como meta a alcanzar y la valoración del avance en el proceso de aprendizaje. Se sugiere seguir precisando los conceptos.

La idea generalizada sobre el indicador de logro es el parámetro que permite valorar el nivel alcanzado por el estudiante durante el proceso para conseguir el logro.

Las respuestas denotan que más que un orientador del proceso propio de los estudiantes, el maestro continúa siendo el guía en el aprendizaje del estudiante, quien determina qué y cómo debe realizarse el proceso educativo. Se percibe que este proceso educativo -que debe visualizarse como el fin de la práctica pedagógica y por lo tanto del maestro--, no se relaciona con el estudiante como un medio de apropiarse de valiosas "herramientas' para su vida, su desarrollo personal y profesional, sino como algo que "debe" hacerse, un requisito. El "educar-se" como parte del proyecto de vida, que incide y se desarrolla con un contexto real y que permite modificarlo desde su conocimiento, puede ser una búsqueda que estamos en capacidad de motivar en los estudiantes, no en un afán voluntarista sino en dirección de acompañar esos proyectos de vida propios de cada uno. La gran pregunta es la factibilidad de una educación para la vida, en un sistema que exige ciertos parámetros incompatibles Porcentajes con esta concepción.

Actividad 18. Escriba un logro y un indicador de logro correspondiente a una temática de la química.

Ejemplos de logro. -Muestra 20 profesores- 


\begin{tabular}{|l|c|}
\hline CATEGORIAS & Porcentajes \\
\hline $\begin{array}{l}\text { a. Valorar la actividad en la vida real o } \\
\text { Cotidiana }\end{array}$ & $20,0 \%$ \\
\hline b. Establecer semejanzas y diferencias & $10,0 \%$ \\
\hline $\begin{array}{l}\text { c. Ampliar los conceptos... para relacionar/ } \\
\text { Interpretar con fenómenos de la vida real }\end{array}$ & $10,0 \%$ \\
\hline $\begin{array}{l}\text { d. Definir el concepto... a través de la } \\
\text { Observación de... }\end{array}$ & $5,0 \%$ \\
\hline $\begin{array}{l}\text { e. Desarrollar habilidades y/o destrezas } \\
\text { Para el trabajo en ciencias }\end{array}$ & $5,0 \%$ \\
\hline $\begin{array}{l}\text { f. Reconocer la importancia de... y valorar } \\
\text { Beneficios y perjuicios }\end{array}$ & $5,0 \%$ \\
\hline $\begin{array}{l}\text { g. Interpretar, analizar e inferir resultados } \\
\text { A partir de la interrelación de variables }\end{array}$ & $10,0 \%$ \\
\hline h. Identificar propiedades, clasificar... & $20,0 \%$ \\
\hline i. Describir la importancia de... & $5,0 \%$ \\
\hline j. Analizar las implicaciones de los aportes... \\
Al desarrollo de... & $10,0 \%$ \\
\hline k. Conocer y explicar situaciones. & $5,0 \%$ \\
\hline
\end{tabular}

\section{Ejemplo de indicador de logro}

-Muestra 20 profesores-

\begin{tabular}{|l|c|}
\hline CATEGORIAS & Porcentajes \\
\hline a. Reconoce el aporte... & $5,0 \%$ \\
\hline b. Realiza mapa conceptual & $5,0 \%$ \\
\hline c. Identifica cálculos y procedimientos & $5,0 \%$ \\
\hline d. Define... & $5,0 \%$ \\
\hline e. Describe con fundamentos y/o actividades... & $5,0 \%$ \\
\hline f. Identifica... & $15,0 \%$ \\
\hline $\begin{array}{l}\text { g. Establece relaciones cualitativas y cuantitativas } \\
\text { Entre... y... para presentar relaciones condicionales }\end{array}$ & $10,0 \%$ \\
\hline h. Clasifica, reconoce, menciona usos... & $10,0 \%$ \\
\hline $\begin{array}{l}\text { i. Diferencia entre... y..., interpreta... diferencia } \\
\text { Características }\end{array}$ & $5,0 \%$ \\
\hline j. Elabora ensayo donde establece aportes de ... & $5,0 \%$ \\
\hline k. Relaciona conceptos... con cotidianidad & $5,0 \%$ \\
\hline I. Interpreta y aplica & $10,0 \%$ \\
\hline m. Propone experiencias para aclarar diferencias & $5,0 \%$ \\
\hline $\begin{array}{l}\text { n. Explica utilizando terminología adecuada (fórmulas, } \\
\text { Conceptos) }\end{array}$ & $15,0 \%$ \\
\hline o. Indaga aportes dentro de un contexto histórico & $5,0 \%$ \\
\hline p. Elabora diagramas & $5,0 \%$ \\
\hline
\end{tabular}

Como vemos las respuestas son muy generales y se refieren a una amplia variedad de aspectos que de una $u$ otra manera siguen llevando una cierta carga tradicional pero que también presentan aspectos de alguna manera novedosos. 
Llegados a este momento los investigadores realizan una cierta evaluación constructiva de todas las respuestas dadas a las diferentes actividades y que fueron consignadas por escrito por los profesores participantes. Hemos compartido las siguientes preocupaciones:

Algunos docentes muestran deficiencias en la competencia básica de lectoescrítura. Esto es preocupante, dado que afecta su comunicación con sus estudiantes y por ende su desempeño docente.

La redacción de casi todas las respuestas a los instrumentos aplicados, muestran un centrado interés en que el proceso educativo se circunscriba al desarrollo de temáticas que el docente estima convenientes. Esto devela el aislamiento respecto a los aspectos sociales, económicos y culturales en los que posteriormente se desempeñarán los ahora estudiantes. Tal vez la evaluación continua - real y efectiva - de aspectos cualitativos y cuantitativos, pueda orientar la pertinencia del trabajo en aulas respecto a la sociedad, que en gran medida debe ser iniciativa del estudiante en una labor de sensibilización que puede favorecer el docente.

Hemos criticado la noción de competencia como "saber hacer en un contexto", que a veces no trasciende a la práctica como lo revela la formulación de logros e indicadores y el cómo se evalúan.

Si la educación pretende favorecer un aprendizaje significativo, lo significativo, en nuestra opinión se alcanza a partir esencialmente de lo actitudinal. A pesar de ser mencionado en las concepciones de los profesores, en las aplicaciones o ejemplos es casi invisible esa trascendencia.

Para aportar a una mejor correlación entre el discurso y las realizaciones prácticas, los investigadores realizan un trabajo de intervención didáctica que permite a los participantes relacionar el modelo evaluativo con el tema de las competencias, los logros y los indicadores a propósito de la educación en química. Para tal efecto preparamos un documente sobre 'Competencias en el área de ciencias naturales' que sirvió de apoyo al estudio de la temática.

Actividad No. 19. En forma individual realizar el estudio del documento: "competencias en el área de ciencias naturales". Haga un análisis crítico a las competencias que allí se señalan como prioritarias. Identifique los logros que se plantean y las posibilidades de evaluación. Critique los ejemplos que se aportan y si se corresponden con lo planteado teóricamente.

Actividad No. 20. En pequeños grupos compartir el trabajo realizado estando muy atentos a una interacción permanente que permita redimensionar lo hecho a nivel individual. En lo posible sacar conclusiones de grupo.

Actividad No. 21. Con la orientación de los investigadores, realizar una plenaria para presentar el trabajo de los grupos.

Este trabajo permitió que la mayoría de los profesores pudieran no solo compartir sus propias interpretaciones sino también recibir retroalimentación del grupo para aclarar, ampliar, precisar y dar ejemplos concretos para el caso de la química. Se hizo hincapié en las dimensiones cognitivas, afectivas, sociales. Se trabajaron actividades específicas de evaluación para el aprendizaje significativo de conceptos; para el manejo de procedimientos a través de situaciones problemáticas y de relaciones química, tecnología 
química y sociedad. Se explicaron con ejemplos las actividades de autorregulación e interregulación como fundamentales para hacer de la evaluación un instrumento de aprendizaje. Para cada caso se trabajaron ejemplos de aplicación analizando siempre críticamente sus coherencias con la teoría.

En estas interacciones surgieron evidencias del avance de los participantes en la transformación de sus ideas sobre la evaluación. En sus análisis aparecen argumentos derivados de concebir la evaluación como instrumento de aprendizaje y no solo como instrumento de constatación terminal del aprendizaje de los estudiantes. Se evidencia una mejor explicitación de las intencionalidades de la educación en química en términos de competencias, logros e Indicadores que se evalúan a través de actividades adecuadas,

Actividad No. 22. Con base en el trabajo realizado, la información aportada por los demás participantes y los documentes elaborados para ampliar las visiones personales, elabore una propuesta de evaluación. Seleccione para tal fin el tema que más le guste y que mejor domine. Prepare una presentación oral ante el grupo.

Actividad No. 22. Plenaria. Realizar la exposición de las propuestas personales y someterlas a la crítica de los participantes. Los investigadores orientaron este trabajo de exposición e intercambio de proyectos evaluativos. Se realizaron filmaciones de las sesiones y se concluyó con una evaluación final del trabajo. Posteriormente los investigadores trabajaron con cada uno de los maestros el análisis de las actividades concretas con el fin de favorecer un proceso de reestructuración de las actividades de acuerdo con el modelo teórico trabajado. Se sugirió a los participantes aplicar en sus instituciones la propuesta y producir un informe sobre este trabajo. Una de las limitaciones que hemos tenido como investigadores ha sido la dificultad práctica de realizar el seguimiento a la puesta en práctica de las propuestas de evaluación lo cual podría darnos elementos adicionales para valorar los resultados en la práctica de la estrategia experimentada.

Corno hemos advertido reiteradamente, nuestra propuesta de evaluación va más allá de "medir" los resultados finales del trabajo pedagógico-didáctico realizado. Por consiguiente no se trata solamente de valorar todo el trabajo a través de la presentación de las propuestas en esta última sesión. Como lo hemos registrado a lo largo de todo el informe, cada actividad del programa-guía, aporta ciertos elementos de juicio para ir valorando los avances y los retrocesos en el proceso de intervención didáctica y se han realizado las acciones más adecuadas para dinamizar el proceso de transformación de las concepciones y acciones de los participantes respecto a la evaluación. De esta manera, estas actividades finales aportan otros elementos adicionales al conocimiento sobre los posibles cambios producidos en los participantes. Desde esta perspectiva incluimos aquí el análisis de las propuestas de evaluación hechas por los maestros.

Los trabajos de los maestros en su mayoría presentan una fundamentación teórica que asigna a la evaluación funciones distintas a las de medida final del aprendizaje de los estudiantes y aceptarla como medio para identificar durante el proceso de enseñanza y aprendizaje los logros y dificultades para tomar los correctivos necesarios.

La mayoría de actividades de evaluación se refieren al aprendizaje de conceptos; las preguntas son de tal naturaleza que para resolverlas no basta con la repetición de los mismos. Es necesario que el estudiante establezca relaciones entre los mismos, realice esquemas, diagramas o mapas conceptuales y acuda a su capacidad de análisis y síntesis para solucionar adecuadamente los interrogantes. 
En este grupo de profesores, en comparación con otros, ha habido una mayor y mejor aplicación del modelo a la hora de proponer actividades que permitan el manejo de aspectos claves del trabajo científico. Esto se corrobora a través del planteamiento de situaciones problemáticas para cuya solución se requiere que los estudiantes: realicen análisis cualitativos del fenómeno en cuestión y precisen sus variables, emitan hipótesis y planteen diferentes alternativas de solución.

Se encuentran algunas pocas actividades referidas a la producción de pensamiento en los estudiantes sobre las implicaciones de la ciencia, la tecnología y la sociedad. Al sugerirles considerar estos aspectos introducen preguntas de esta naturaleza sin mucha dificultad.

Se encuentran muy pocas actividades de autorregulación e interregulación cuya realización permitiría a los estudiantes reflexionar y tomar conciencia de sus propios avances, carencias o dificultades, permitiendo al profesor aportar retroalimentación en el mismo momento en que los estudiantes están realizando las actividades evaluativas.

Quizá por la naturaleza misma de la tarea planteada a los maestros, aparecen en las propuestas escasas referencias a la evaluación como instrumento de mejora de la docencia pues no se plantean explícitamente actividades que permitan conocer la opinión de los estudiantes respecto al trabajo del profesor en cuanto a la calidad de sus metodologías y materiales didácticos empleados; tampoco aparecen referencias a la evaluación del clima del aula y en general del clima institucional.

\section{CONCLUSIONES}

\section{Sobre las ideas previas de los profesores de química y su evolución}

Con este grupo de maestros se obtienen inicialmente respuestas más próximas al modelo de evaluación como instrumento de aprendizaje y mejora de la docencia ya que se hace referencia a los fines de la evaluación no sólo para conocer lo que ocurre con los estudiantes sino también lo que pasa con los profesores y las metodologías utilizadas. Los profesores manifestaron en un alto porcentaje que la evaluación es útil para medir tanto el desempeño docente como las metodologías empleadas. También es significativo el interés por revisar la reestructuración de conceptos en los estudiantes. De este modo se percibe la evaluación como un instrumento de ayuda para favorecer aprendizajes significativos.

Consideran que la evaluación debe ser continua y orientada a favorecer aprendizajes significativos a través de la resolución de problemas; plantean que la evaluación puede generar un acompañamiento en los procesos de aprendizaje tanto para estudiantes como para maestros. También se destaca la idea de la evaluación no sólo individual sino grupal, y la autoevaluación como características importantes a tener en cuenta.

Consideran importante evaluar los conocimientos teóricos, las habilidades rnetodológicas y las actitudes.

Identifican los principales fallos de las evaluaciones tradicionales; se refieren a la presencia de preguntas de cede memorístico, cerradas que inducen a resoluciones de tipo mecánico. Sin embargo, cuando surgieron cambios para mejorar el examen sus preferencias no se apartan de las preguntas tradicionales. 
Sugieren evaluar en términos de situaciones problemáticas para cuya comprensión y resolución se requeriría no sólo de manejos conceptuales sino también procedimentales y actitudinales. Consideran necesario tener en cuenta las ideas previas de los estudiantes. Esto indica que la preocupación en la evaluación consiste en favorecer un aprendizaje significativo lo cual nos aporta evidencias sobre los cambios que se van logrando en la comprensión de los aspectos a evaluar.

La mayoría considera que el concepto de competencia hace referencia a un saber hacer en un contexto". Las competencias más evaluadas son la interpretativa y la argumentativa. Para su evaluación se sugiere el planteamiento de situaciones problemáticas, las intervenciones verbales, la elaboración de resúmenes, ensayos y la realización de lecturas. Otras propuestas como: juegos, trabajos en grupo, realización de mapas, cuadros, criticas a lecturas, planteamiento de hipótesis, y diseño experimental, permiten una mayor motivación, sensibilización y concientización de los estudiantes respecto a su aprendizaje y además exigen cambios en los imaginarios docentes.

Los logros son momentos de desarrollo y los indicadores son los estados en los cuales se encuentra el estudiante dentro de esos momentos de desarrollo. Logro es el proceso que tiene un fin e indicador es un indicio del proceso. Los logros son objetivos a cumplir y los indicadores sontos criterios que miden lo que se alcanzó o no. El indicador de logro es el parámetro que permite valorar el nivel alcanzado por el estudiante durante el proceso para conseguir el logro.

\section{Sobre los cambios evidenciados de los profesores de química en proyectos de aula}

La mayoría de los trabajos escritos y las sustentaciones orales hechas por los profesores de maestría presentan una fundamentación teórica sobre la evaluación que aporta evidencias acerca de la concepción de evaluación corno instrumento de aprendizaje. Las finalidades descritas se metieren básicamente a posibilitar a través de la evaluación la toma de conciencia de los avances y dificultades de los estudiantes para mejorar la calidad de sus aprendizajes; se trata entonces de utilizar la evaluación corno instrumento de ayuda real y efectiva y no simplemente de constatación terminal de los logros de los estudiantes, lo anterior no quiere decir que la evaluación pierda su valor como indicador de aprendizaje; esta función sigue siendo reconocida por los profesores corno fundamental, pero se acepta que debe ser permanente para aportar en el momento justo, las orientaciones que el buen aprendizaje requiere.

La mayoría de las propuestas han planteado en las diferentes temáticas, actividades de evaluación que superan las tradicionales preguntas de repetición mecánica de conceptos o definiciones. Se encuentran actividades que enfatizan en el manejo significativo de conceptos lo cual implica que los estudiantes no las pueden resolver a través de la simple repetición de los conocimientos, requieren para tal efecto, superar sus ideas previas, establecer relaciones nuevas entre los conceptos, realizar esquemas, diagramas o mapas conceptuales.

En menor proporción pero en muchas de las propuestas aparecen también actividades que además de poner en juego el aprendizaje conceptual, requieren el manejo de metodologías mas próximas a las empleadas por los científicos y que tiene que ver con: el análisis cualitativo de situaciones problemáticas, la identificación de variables, la emisión de hipótesis, la elaboración de estrategias ce resolución, su resolución y el análisis de los 
resultados. Este grupo utiliza para favorecer el cambio metodológico actividades referidas a situaciones problemáticas

También incluyen las propuestas actividades que exigen al estudiante la producción de análisis críticos de las relaciones entre la química, la tecnología química y los contextos sociales donde los procesos químicos ocurren y sus impactos positivos y negativos. Esto lo realizan a través de lecturas sobre problemas ambientales derivados del uso de procesos de producción química.

Las propuestas contienen pocas actividades de autorregulación e interregulación cuya realización permitiría a los estudiantes reflexionar y tomar conciencia de sus propios avances, carencias o dificultades, permitiendo al profesor aportar retroalimentación en el mismo momento en que los estudiantes están realizando las actividades evaluatívas. Al sugerirles a los estudiantes tener en cuenta estas relaciones logran incorporarías a las actividades de evaluación.

No aparecen en las propuestas referencias a la evaluación como instrumento de mejora de la docencia pues no se plantean explícitamente actividades que permitan conocen la opinión de los estudiantes respecto al trabajo del profesor en cuanto a la calidad de sus metodologías y materiales didácticos empleados; tampoco aparecen referencias a la evaluación del clima del aula y en general del clima institucional

\section{Sobre la estrategia pedagógica-didáctica experimentada}

Como se ha podido verificar el tema de la evaluación requiere de un tratamiento a profundidad pon parte de los profesores con miras a lograr transformaciones de fondo tanto en las concepciones como en las acciones; este asunto es vital en los procesos de formación inicial y permanente de docentes si se quiere aportar a la construcción de una nueva cultura respecto al conocimiento y al compromiso individual y colectivo de mejoramiento social.

Los momentos centrales del programas-guía de actividades son:

- Explicitación de ideas previas sobre: funciones, características, momentos, formas de la evaluación que realizan.

- Análisis crítico de las ideas previas a la luz del modelo de evaluación como instrumento de aprendizaje y mejora de la docencia.

- Cuestionamiento a la idea de "objetividad" de las evaluaciones.

- Análisis critico a los exámenes realizados por los participantes.

- Introducción crítica del modelo de evaluación como instrumento de aprendizaje y mejora de la docencia.

- Aplicación del modelo a la evaluación de temáticas especificas.

- Ideas previas sobre los conceptos de competencia, logro e indicador.

- Introducción crítica de la propuesta de evaluación por competencias y su relación con el modelo evaluativo. 
- Aplicación de los conceptos a la evaluación de temáticas específicas.

- Experimentación en el aula de las propuestas realizadas por los participantes.

\section{REFERENCIAS BIBLIOGRÁFICAS}

ALONSO, M. (1990) Propuesta de evaluación en Física y análisis de la evaluación habitual. Tesis de master. Universidad de Valencia. España.

ALONSO, M., GIL, D.,Y MARTÍNEZ TORREGROSA, J. (1992) Concepciones Espontáneas de los profesores de Ciencias sobre la Evaluación : Obstáculos a superar y propuestas de replanteamiento. Revista de Enseñanza de la Física, 5 (2), pp. 18-38.

ALONSO, M., (1994 ). La Evaluación en la Enseñanza de la Física. Tesis de Doctorado. Universidad de Valencia.

AUSUBEL, D., 1978. Sicología Educativa: Un punto de vista cognoscitivo (Trillas: México).

BELTRAN, H., LAVERDE, S, TAMAYO, (1997). Las Interacciones Ciencia! Tecnología ¡Sociedad en el Proceso de Formación de Docentes. Trabajo de grado. Departamento de Química. U.P.N.

PAYA, J., 1991. Los trabajos prácticos en la enseñanza de la Física y la Química. Un análisis crítico y una propuesta fundamentada. Tesis Doctoral. Universidad de Valencia.

PERAFÁN, A. REYES, L., SALCEDO, L., 2001. Acciones y creencias. Tomo II. Universidad Pedagógica Nacional. Editorial ARFO. Bogotá.

REYES L., SALCEDO L., PERAFAN, A., 2001. Acciones y creencias. Tomo IV. Análisis e interpretación de creencias de docentes en biología y ciencias naturales. Universidad Pedagógica Nacional. Editorial ARFO. Bogotá.

RINCON, L., 1996. Diagnóstico sobre la Evaluación Realizada pon los profesores de Química del Departamento de Boyacá. Tesis de Maestría en docencia de la Química. U.P.N. Convenio UPTC - UPN.

SALCEDO, L 1994 Concepciones de los profesores de Química de secundaria sobre las prácticas de laboratorio. IX Congreso Colombiano de Química. Santa Marta. Memorias.

SALCEDO L., JESSUPM., PLAMENN., 1996 Evaluación en la Enseñanza de las Ciencias. Educación y Cultura No 572, Pág. 17-23.

SALCEDO L., VILLARREAL M., 1999. Concepciones y Acciones de los Profesores de Química sobre la Evaluación. Revista Educación y Pedagogía No. 25 Vol. XI. Pág. 177207.

SAlCedo, L., ReYeS, L., PeRAFÁN, A., 2001. Acciones y creencias. Tomo III. Universidad Pedagógica Nacional. Editorial ARFO. Bogotá. 
SCHIBECl, R. 1984. Attítudes te Science An update. Studies in science education, 11, pp. 26- 59.

VALCARCEL N., 1996.Estudio descriptivo sobre la evaluación en la escuela de Química de la Universidad Pedagógica y tecnológica de Colombia. Tesis de maestría.

VILCHES A. ,1 993 Las interacciones CTS en la enseñanza de las ciencias fisicoquímicas. Tesis doctoral. Valencia. España. 